\title{
Operating Windows of Pebble Divertor
}

\author{
K. Matsuhiro, M. Isobe, Y. Ohtsuka, Y. Ueda and M. Nishikawa \\ Graduate School of Engineering, Osaka University, Osaka, Japan \\ e-mail contact of main author: matuhiro@ppl.eng.osaka-u.ac.jp
}

\begin{abstract}
A marked feature of the pebble divertor is an effect by use of functional multi-layer coated pebble, which consists of a surface plasma facing layer, an intermediate tritium permeation barrier layer, and a kernel for heat removal. The dimensions, structure and the irradiation conditions of pebbles are the important issues for the development of the pebble divertor. From the view point of resistance of the induced thermal stress, the pebble is taken as small as possible in size. On the other hand, from the view point of the pumping performance, the suitable irradiation temperature range of the surface layer of pebble was estimated from the experiments and the numerical analysis. The pumping process enhanced by dynamic retention is available to extend the higher allowable irradiation temperature range from $900 \mathrm{~K}$ to $1100 \mathrm{~K}$. As taking the temperature rise limitation due to pumping effect and the fractural strength due to the induced thermal stress limitation, it was found that the diameter of the pebble is possible to be $1-2 \mathrm{~mm}$ in about $20 \mathrm{MW} / \mathrm{m}^{2}$ for the $\mathrm{SiC}$ kernel and 2-3 $\mathrm{mm}$ in less than $30 \mathrm{MW} / \mathrm{m}^{2}$ for the graphite kernel.
\end{abstract}

\section{Introduction}

A pebble divertor [1,2] is developed for the application of high power density fusion reactors. In this system, a large number of small refractory pebbles are falling in the divertor space. A marked feature of our pebble divertor system is to use functional multi-layer pebble (cf. divertor pebbles). The incident heat and particle loading to the divertor are taken away by the divertor pebbles. Because the irradiation periods of fast falling pebbles are very short, the surface coatings can be applied to the divertor pebble without erosion problems. The multi-layer pebble consists of a kernel (made of SiC or carbon), the plasma facing layer (CVD coating graphite), and the intermediate layer (CVD coating SiC). Each layer has specific functions as follows. The surface plasma facing layer controls plasma-wall interaction. The kernel plays an important role in mechanical and thermal properties of the whole pebble. The intermediate layer as a tritium permeation barrier prevents bulk tritium retention. By these functions of multi-layer pebbles and the external pebble processing systems, the pebble divertor system has the following features, (1) pebble conveying without MHD problem, (2) continuous wall pumping, (3) both pumping function and low bulk tritium retention are realized by tritium barrier layer, (4) continuous replacement of eroded surface components. Furthermore, the fabrication technology of multi-layer coated ceramic pebbles has been developed for High Temperature Gas-Cooled Fission Reactor.

Some functions of the pebble divertor mainly depend on the irradiation temperature of the multi-layer pebble. Fig. 1 shows that the temperature cycle of the surface of a divertor pebble in the pebble divertor system. The divertor pebbles at initial temperature (cf. inlet temperature) are heated up to the irradiation temperature in the strike zone of the divertor. After the irradiation, the pebbles are transported to the regeneration stage and are further heated up to regeneration temperature to desorb the retained fuel gas and impurities. Then, the regenerated pebbles are cooled down and are returned to the divertor. The pumping performance is affected by the irradiation temperature and regeneration temperature. So, the irradiation temperature must be in appropriate range for good pumping performance. On the design of pebble divertor system, the size, or heat capacity of the divertor pebble and the falling speed of it control the irradiation temperature. Because the pebble size is also restricted 


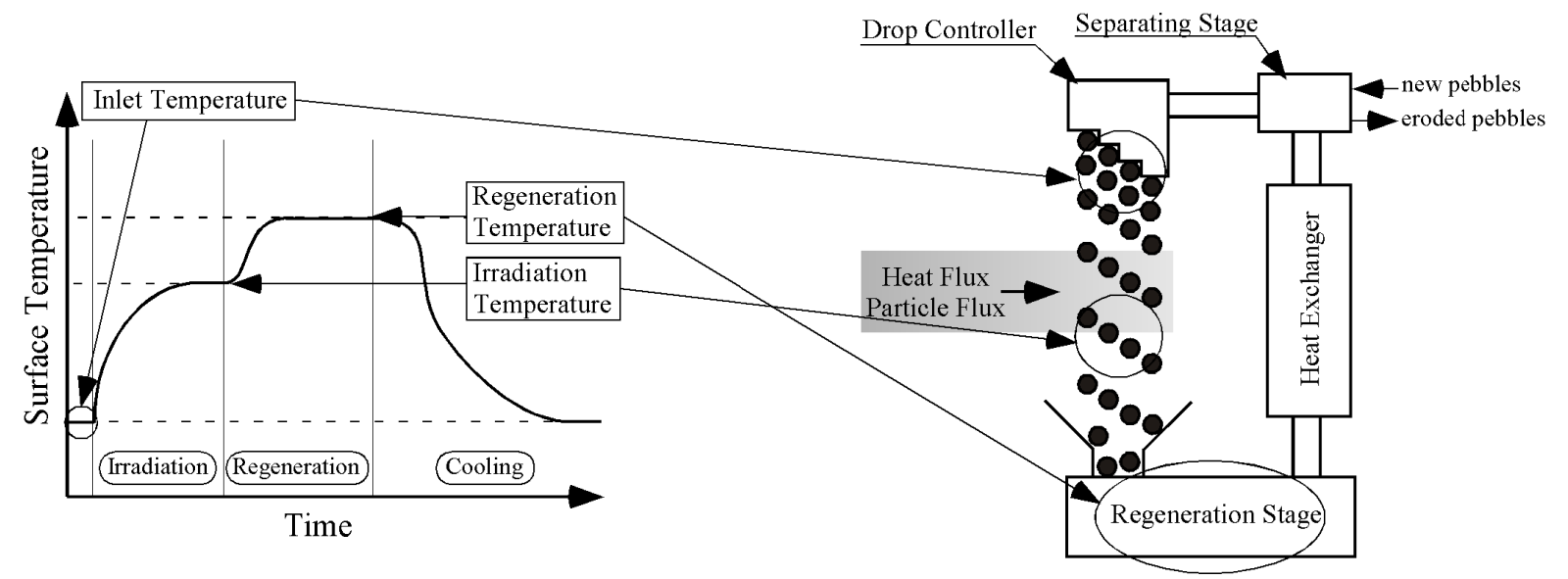

Fig. 1. The cycle of the divertor pebble and the temperature of the pebble.

by the thermal stress at the given heat load, the dimensions of the divertor pebble is determined by both heat load and pumping specifications.

In this paper, the window of the irradiation temperature was estimated from the relation between irradiation temperature and the pumping capacity. For this purpose, we performed experiments that the hydrogen release from the graphite samples just after the high flux hydrogen beam irradiation was measured at the irradiation temperature of $573-973 \mathrm{~K}$. Then the hydrogen release at the irradiation temperature over $973 \mathrm{~K}$ was calculated by solving the mass balance equations. From the window of the irradiation temperature for the pumping and the compressive fractural strength of the kernel material, the relation between the pebble diameter and the allowable heat load was estimated by assuming the irradiation period determined from the width of the strike zone and the falling speed of the divertor pebbles.

\section{Irradiation Temperature Window for Fuel Gas Pumping}

Recently, the divertor systems which have the pumping function using the wall pumping process were proposed [3, 4]. In these systems, carbon based material were selected as plasma facing wall. Because hydrogen retention in carbon decreases with increasing in the temperature [5], the difference between the saturated amount of retained hydrogen at the irradiation temperature and that at the regeneration temperature is the pumping capacity. In this wall pumping process, the pumping capacity is increasing with decreasing of the irradiation temperature. Therefore, the irradiation temperature needs to be low for good pumping performance, and the allowable heat flux or irradiation time is needed to be lower or shorter respectively.

In the pebble divertor, another wall pumping process is considered. Under the high particle flux irradiation such as particle loading on the divertor plate, graphite retains more hydrogen than the static amount obtained from low flux ion beam experiments. The excess amount of dynamically retained hydrogen is transiently released just after the irradiation. By using this transient gas desorption as a part of regeneration process, the pumping capacity will become larger at high temperature and the irradiation temperature window can be extended.

For estimating the pumping process using the transient hydrogen release, the hydrogen release just after high particle flux irradiation was measured by using the experimental device, which consisted of the high flux beam source [6], the irradiation chamber and the TDS (Thermal 


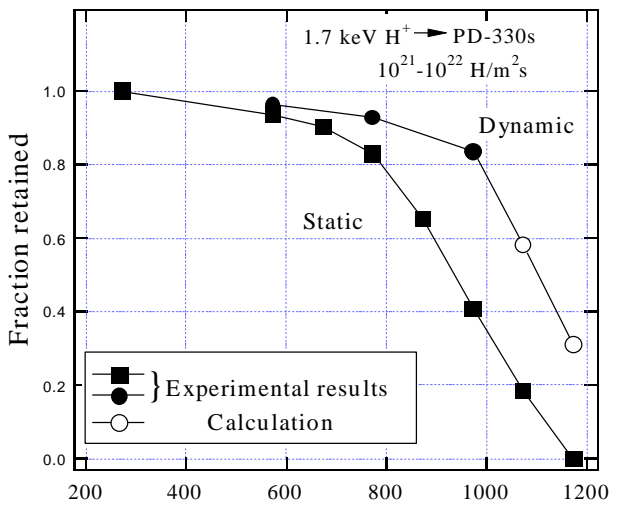

Fig. 2. Temperature depehimenerature rétained hydrogen in graphite.

Desorption Spectroscopy) chamber. In the experiment, the sample was irradiated by the pulsed hydrogen beam $\left(5 \mathrm{keV} \mathrm{H}_{3}{ }^{+}(1.7 \mathrm{keV}\right.$ per $\left.\mathrm{H})\right)$ with maximum particle flux of $1 \times 10^{22}$ $/ \mathrm{m}^{2}$ s. After the irradiation, the sample was moved to the TDS Chamber in $1 \mathrm{sec}$ and the transient hydrogen release was measured in the TDS chamber.

From the experimental results [7], the transient hydrogen was spontaneously released from 5 $\mathrm{sec}$ to $55 \mathrm{sec}$ after the irradiation. Therefore it was found that the amount of dynamically retained hydrogen in graphite under the high flux irradiation is larger than the static retention. The amount of the transient hydrogen release is increasing with increasing of the irradiation temperature. From the experimental result, the temperature dependence of fraction retained hydrogen in graphite was calculated by using the mass balance equations model [8].

Fig. 2 shows the temperature dependence of the dynamically and statically retained hydrogen in graphite from the experimental results. The rectangular plot in the static process represents the residual hydrogen at $200 \mathrm{sec}$ after the irradiation at given temperature, and the circle plot in the dynamic process represents the retained hydrogen at $5 \mathrm{sec}$ after the high particle flux irradiation. The dynamic process over $973 \mathrm{~K}$ are estimated from the calculation by solving the mass balance equations. As shown in Fig. 2, the pumping capacity is enhanced to be a large value equivalent to the value at $100-200 \mathrm{~K}$ lower temperature in the static process. The heating power for raising the temperature of the divertor pebble to the regeneration temperature is also saving in relatively small input. The pumping capacity required by ITER will be attained in the dynamic process at $1100 \mathrm{~K}$. So, the irradiation temperature should be below $1100 \mathrm{~K}$.

\section{Appropriate diameter of the divertor pebble}

In our previous work [1], it was shown that the maximum allowable heat loads to the divertor pebbles are determined by the induced thermal stress. Therefore, the diameter of pebble is restricted in high heat flux divertor. For example, a pebble with a graphite kernel must be smaller than $2 \mathrm{~mm}$ in diameter in $30 \mathrm{MW} / \mathrm{m}^{2}$ of heat load. However, the lower limit of the diameter of a pebble is determined by the irradiation temperature related with pumping capacity. For estimating the diameter of pebble, the thermal stress and the surface temperature rise were calculated in the conditions of heat load of 10,20 and $30 \mathrm{MW} / \mathrm{m}^{2}$, which is distributed uniformly on the surface of the divertor pebble. In this calculation, we assumed that the width of the strike zone was $0.1 \mathrm{~m}$, and the divertor pebbles were dropped from $1 \mathrm{~m}$ above the strike zone. Therefore, the irradiation period was about $23 \mathrm{~ms}$. 

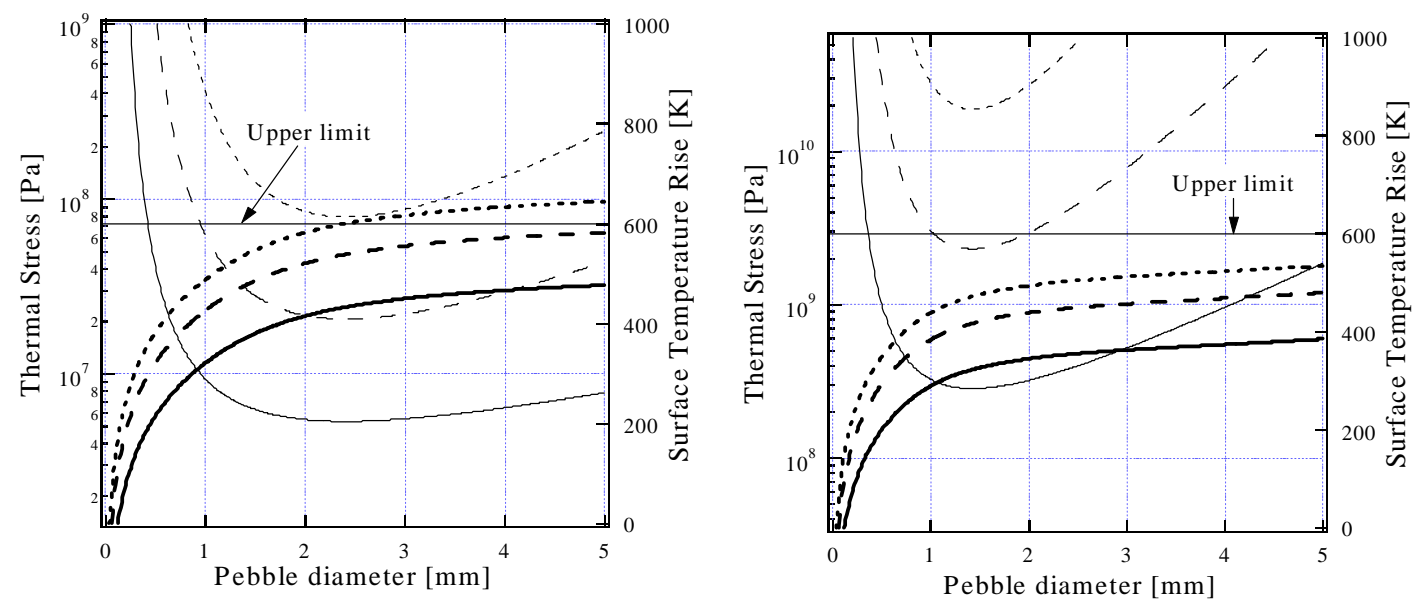

\begin{tabular}{|c|c|}
\hline Thermal Stress & Surface Temperature Rise \\
\hline$-10 \mathrm{MW} / \mathrm{m}^{2}$ & $-10 \mathrm{MW} / \mathrm{m}^{2}$ \\
$--20 \mathrm{MW} / \mathrm{m}^{2}$ & $--20 \mathrm{MW} / \mathrm{m}^{2}$ \\
$\cdots--30 \mathrm{MW} / \mathrm{m}^{2}$ & $--30 \mathrm{MW} / \mathrm{m}^{2}$ \\
\hline
\end{tabular}

(a)graphite kernel

(b)SiC Kernel

Fig. 3.Pebble diameter dependence of the induced thermal stress and the surface temperature rise.

Fig. 3 shows the pebble diameter dependence of the thermal stress and the surface temperature rise for the pebble with graphite (a) and $\mathrm{SiC}$ (b) kernel. The upper limit line for the thermal stress is the compressive fractural strength of graphite and $\mathrm{SiC}$. For estimating the upper limit line of the surface temperature rise, we assumed that the inlet temperature was 500 $\mathrm{K}$. From the irradiation temperature window below $1100 \mathrm{~K}$ in section 2, the upper limit of the surface temperature rise was decided to $600 \mathrm{~K}$.

For graphite kernel, the diameter of the pebble is limited by the surface temperature rise and the thermal stress over $20 \mathrm{MW} / \mathrm{m}^{2}$. At $30 \mathrm{MW} / \mathrm{m}^{2}$, the pebble divertor system cannot satisfy the pumping requirement, because the surface temperature rise is over the upper limit line. Therefore, the allowable heat load is less than $30 \mathrm{MW} / \mathrm{m}^{2}$ and the pebble diameter for graphite kernel should be between 2 and $3 \mathrm{~mm}$ in diameter in the condition.

For SiC kernel, the thermal stress is below the upper limit line at all cases because of the high compressive fractural strength of SiC. However, the surface temperature rise of $\mathrm{SiC}$ kernel is larger than that of graphite kernel at 20 and $30 \mathrm{MW} / \mathrm{m}^{2}$ because of the low heat conductivity and capacity of SiC. Therefore the diameter of the divertor pebble of SiC kernel is limited only by the surface temperature rise, and the allowable heat load was about $20 \mathrm{MW} / \mathrm{m}^{2}$. The diameter of pebbles with a SiC kernel should be between 1 and $2 \mathrm{~mm}$ in the heat flux of 20 $\mathrm{MW} / \mathrm{m}^{2}$. By decreasing the irradiation period for $\mathrm{SiC}$ kernel with the high compressive fractural strength, the allowable heat load can be increased to open the operating windows wide.

\section{Summary}

For estimating the window of the irradiation temperature from the relation between irradiation temperature and the pumping performance, the hydrogen release from the graphite samples just after the high flux hydrogen beam irradiation was measured at the irradiation temperature of $573-973 \mathrm{~K}$. Then the hydrogen release at the irradiation temperature over $973 \mathrm{~K}$ was calculated by solving the mass balance equations. The experimental and calculated results can be summarized as follows: 
- By using dynamic retention under the high particle flux irradiation, the pumping capacity is enhanced to be a large value equivalent to the value at $100-200 \mathrm{~K}$ lower temperature in the static process.

- To obtain appropriate pumping capacity, the irradiation temperature should be below $1100 \mathrm{~K}$.

The operating windows evaluated by the pebble diameter and the allowable heat load on the graphite and $\mathrm{SiC}$ pebble are described as follows:

- For graphite kernel, the allowable heat load is less than $30 \mathrm{MW} / \mathrm{m}^{2}$ and the diameter of the pebble should be between 2 and $3 \mathrm{~mm}$ in diameter in the condition.

- For SiC kernel, the diameter of the divertor pebble of $\mathrm{SiC}$ kernel is limited only by the surface temperature rise, and the allowable heat load was about $20 \mathrm{MW} / \mathrm{m}^{2}$.

- The diameter of pebbles with a SiC kernel should be between 1 and $2 \mathrm{~mm}$ in the heat flux of $20 \mathrm{MW} / \mathrm{m}^{2}$.

- By decreasing the irradiation period for $\mathrm{SiC}$ kernel with the high compressive fractural strength, the allowable heat load can be increased to open the operating windows wide.

\section{References}

[1] M., Isobe, et al., "Multilayer pebbles for application to new divertor systems", Nuclear Fusion, 40 (2000) 647-651.

[2] M., Isobe, et al., "Steady state wall pumping performance of pebble drop divertor", Journal of Nuclear Materials, 258-263 (1998) 745-749.

[3] A., Sagara, et al., "Design of carbon sheet pump for LHD and Demonstration of hydrogen pumping", Journal of Nuclear Materials, 220-222 (1995) 627-630.

[4] Y., Hirooka, et al., "Steady-State Impurity Control, Heat Removal and Tritium Recovery by Moving-Belt Plasma-Facing Components", Proceedings-Symposium-onFusion-Engineering 2 (1998)906..

[5] W. Möller, "Hydrogen trapping and transport in carbon", Journal of Nuclear Materials, 162-164 (1989) 138-150.

[6] M., Nishikawa, et al., "Development of high current density neutral beam injector with a low energy for interaction of plasma facing materials", Fusion Engineering and Design, 16 (1991) 351-356.

[7] K. Matsuhiro, et al., "Pumping Performance of Pebble Drop Divertor", Fusion Engineering (Proc. ISFNT-5 1999) (currently publishihg).

[8] K. Matsuhiro, et al., "Transient Hydrogen Release from Graphite just after High Flux Beam Irradiation", Journal of Plasma and Fusion Research (Proc. 5th Int. Toki Conf. 2000) (currently publishihg). 